\title{
Implant isolation of Zn-doped GaAs epilayers: Effects of ion species, doping concentration, and implantation temperature
}

\author{
Prakash N. K. Deenapanray, ${ }^{\text {a) }}$ Q. Gao, and C. Jagadish \\ Department of Electronic Materials Engineering, Research School of Physical Sciences and Engineering, \\ The Australian National University, Canberra ACT 0200, Australia
}

(Received 27 January 2003; accepted 4 March 2003)

\begin{abstract}
The electrical isolation of $\mathrm{Zn}$-doped GaAs layers grown by metalorganic chemical vapor deposition was studied using $\mathrm{H}, \mathrm{Li}, \mathrm{C}$, and $\mathrm{O}$ ion implantation. The ion mass did not play a significant role in the stability of isolation, and a similar activation energy of $\sim(0.63 \pm 0.03 \mathrm{eV})$ was obtained for isolation using either $\mathrm{H}$ or $\mathrm{O}$ ions. Furthermore, the isolation was stable against isochronal annealing up to $550^{\circ} \mathrm{C}$ as long as the ion dose was $2-3.5$ times the threshold dose for complete isolation, $D_{\text {th }}$, for the respective ion species. By studying the thermal stability and the temperature dependence of isolation, we have demonstrated the various stages leading to the production of stable isolation with the increasing dose of $2 \mathrm{MeV} \mathrm{C}$ ions. For ion doses less than $0.5 D_{\text {th }}$, point defects which are stable below $250^{\circ} \mathrm{C}$ are responsible for the degradation of hole mobility and hole trapping. The stability of isolation is increased to $\sim 400{ }^{\circ} \mathrm{C}$ for a dose $D_{\text {th }}$ due to the creation of defect pairs. Furthermore, the hopping conduction mechanism is already present in the damaged epilayer implanted to $D_{\text {th }}$. Higher order defect clusters or complexes, such as the arsenic antisite, $\mathrm{As}_{\mathrm{Ga}}$, are responsible for the thermal stability of implantation isolation at $550{ }^{\circ} \mathrm{C}$. The substrate temperature $\left(-196-200{ }^{\circ} \mathrm{C}\right)$ does not have an effect on the isolation process further revealing that the stability of isolation is related to defect clusters and not point-like defects. An average number of eight carbon ions with energy of $2 \mathrm{MeV}$ are required to compensate 100 holes, which provides a general guideline for choosing the ion dose required for the isolation of a GaAs layer doped with a known $\mathrm{Zn}$ concentration. A discussion of the results on the implantation isolation of $p$-GaAs previously reported in the literature is also included. (C) 2003 American Institute of Physics. [DOI: 10.1063/1.1569664]
\end{abstract}

\section{INTRODUCTION}

Ion implantation of compound semiconductor materials is routinely used in the fabrication of electronic and optoelectronic devices. For instance, it is mainly used for creating doped layers (i.e., conductive $n$ - or $p$-type layers) in otherwise semi-insulating material. On the other hand, ion implantation can be used to render a conducting layer highly resistive (i.e., isolating) through the creation of free carrier trapping centers, and hence the term implant isolation. ${ }^{1,2}$ Electrical isolation of III-V semiconductors is now perhaps the most important application of ion implantation in this group of materials, due to the widespread use of epitaxial growth techniques for obtaining doped layers. Regarding GaAs integrated circuit technology, implantation isolation has been widely used in the fabrication of a wide range of planar electronic devices, including heterojunction bipolar transistors (HBTS), impact avalanche transit time (IMPATT) diodes, and metal-semiconductor field effect transistors (MESFETS) (see Ref. 1 for a review). Furthermore, implant isolation is also commonly used to manufacture optoelectronic devices, such as the gain guided, graded index separate confinement heterostructure (GRINSCH) GaAs/AlGaAs quantum well laser, ${ }^{1,3}$ and the vertical cavity surface emitting lasers (VCSELs). ${ }^{4-6}$

\footnotetext{
${ }^{a)}$ Electronic mail: pnk109@ rsphysse.anu.edu.au
}

Implant isolation is achieved by creating free carrier trapping sites related to either radiation-induced damage, i.e., defect isolation, or to the implanted species, i.e., chemical isolation. ${ }^{1,2}$ Compared to the chemical isolation of thick layers which requires multiple implantation steps to create a uniform distribution of the chemical species, defect isolation using light ions $\left(\mathrm{H}^{+}, \mathrm{He}^{+}, \mathrm{B}^{+}, \mathrm{Li}^{+}, \mathrm{C}^{+}, \mathrm{O}^{+}\right.$, or $\left.\mathrm{F}^{+}\right)$can be achieved in a single implantation step. Defect isolation is, therefore, the more attractive alternative for device fabrication purposes. Furthermore, implantation produces both the superior electrical isolation and preserves the semiconductor surface planarity compared to mesa etching.

The electrical isolation of ion-implanted $n$-type GaAs has received much attention in the past few years. ${ }^{7-13}$ Most of those studies have been driven by the need to enhance the current understanding of the physical mechanisms that underlie defect isolation. The results from the comprehensive investigation of de Souza and coworkers ${ }^{7-10}$ suggest that antisite defects created by replacement collisions and/or their defect complexes are responsible for free carrier trapping in GaAs. Comparatively fewer investigations of implant isolation of $p$-type GaAs have been performed. ${ }^{14-16}$ Those studies have utilized the relatively light ions such as $\mathrm{H}$ and $\mathrm{He}$, and used either $\mathrm{C}$ or $\mathrm{Mg}$ as a $p$-type dopant. ${ }^{14-16}$ In some investigations the conductive $p$-type layers were formed in semiinsulating GaAs substrates by $\mathrm{C}$ or $\mathrm{Mg}$ ion implantation followed by annealing to activate the dopant atoms and to remove implantation-induced damage. ${ }^{15,16}$ Since ion implan- 
TABLE I. Summary of the ion implantation conditions used in this work. Calculated values of projected ion ranges $\left(R_{p}\right)$ and the integrated number of replacements, $N_{d}$, produced within a $500 \mathrm{~nm}$ thick GaAs layer by each ion using TRIM code are also shown.

\begin{tabular}{ccccccc}
\hline \hline Ion & $\begin{array}{c}\text { Energy } \\
(\mathrm{MeV})\end{array}$ & $\begin{array}{c}\text { Doses } \\
\left(10^{11} \mathrm{~cm}^{-2}\right)\end{array}$ & $\begin{array}{c}\text { Beam flux } \\
\left(10^{11} \mathrm{~cm}^{-2} \mathrm{~s}^{-1}\right)\end{array}$ & $\begin{array}{c}\text { Irradiation } \\
\text { temperature }\left({ }^{\circ} \mathrm{C}\right)\end{array}$ & $R_{p}(\mu \mathrm{m})$ & $N_{d}\left(/\right.$ ion/ $\left.\mathrm{cm}^{2}\right)$ \\
\hline${ }^{1} \mathrm{H}$ & 0.1 & $2-750$ & 1.8 & 20 & 0.77 & 2.27 \\
${ }^{1} \mathrm{H}$ & 0.6 & $1-3600$ & 1.8 & 20 & 5.78 & 0.34 \\
${ }^{7} \mathrm{Li}$ & 2.0 & $1.0-140$ & 1.6 & 20 & 4.01 & 8.00 \\
${ }^{12} \mathrm{C}$ & 2.0 & $0.2-700$ & 0.60 & $-196-200$ & 2.02 & 41.50 \\
${ }^{16} \mathrm{O}$ & 1.2 & $0.1-20$ & 0.15 & 20 & 1.20 & 152.00 \\
\hline \hline
\end{tabular}

tation produces a nonuniform doping profile, ${ }^{15,16}$ and because practical devices are manufactured by epitaxial growth, it may be more reasonable to investigate implant isolation processes in uniformly doped $p$-type epilayers. ${ }^{14}$ The results reported in Refs. 14-16 have provided much insight into both the mechanism and thermal stability of implant isolation in $p$-GaAs, and will be referred to in the discussion of the results reported here.

In this study, the implant isolation of $p$-GaAs epilayers grown by metalorganic chemical vapor deposition is investigated for a wide range of ion species and free carrier concentrations of epilayers. The implantation temperature is shown to have a negligible effect on electrical isolation and its thermal stability. The thermal stability of isolation for samples doped to different $\mathrm{Zn}$ concentrations and implanted with the different ion species is also discussed alongside the mechanism of defect isolation in $p$-GaAs. In order to gain more insight into the mechanism of the isolation process, we have extracted activation energies by performing extensive temperature dependent measurements of the sheet resistance of ion implanted samples both before and after postimplantation annealing to reveal the stages in the buildup of a stable implant isolation of $p$-GaAs.

\section{EXPERIMENTAL PROCEDURE}

The epilayers used were grown at $650{ }^{\circ} \mathrm{C}$ by lowpressure metalorganic chemical vapor deposition (MOCVD) on semi-insulating (SI) GaAs (100) substrates. A $200 \mathrm{~nm}$ thick nominally undoped buffer layer was grown on the SI substrate prior to the growth of the $500 \mathrm{~nm}$ thick Zn-doped layers at 76 Torr. Epitaxial layers doped with $9 \times 10^{16}, 4.5$ $\times 10^{17}$, or $1.8 \times 10^{18} \mathrm{Zn} / \mathrm{cm}^{3}$ were grown. The free carrier concentration of an epilayer was obtained from electrochemical capacitance-voltage (ECV) measurements. Resistors of rectangular geometry $\left(\sim 6 \times 4 \mathrm{~mm}^{2}\right)$ were cleaved from wafers, and ohmic contacts were formed by manually applying indium (In) strips on the two opposite sides of each resistor followed by sintering at $250{ }^{\circ} \mathrm{C}$ for 2 min under $\mathrm{Ar}$ flow. Besides forming ohmic contacts for sheet resistance measurements, the In strips also masked the underlying epilayer from implantation.

These resistors were irradiated in the Australian National University (ANU) 1.7 MV tandem accelerator (NEC, 5SDH-4) using 0.6-2 MeV ion of different species and at different substrate temperatures. The $0.1 \mathrm{MeV} \mathrm{H}$ ion implantation was performed in a ANU-built low-energy ion im- planter. The ion implantation conditions are summarized in Table I. The ion energies were chosen to place the damage peak deep into the SI GaAs substrate (i.e., produce a quasiuniform damage profile in the doped epilayers), as seen from the calculated values of projected ion ranges using transport of ions in matter (TRIM) code simulations. ${ }^{17}$ An exception is the $0.1 \mathrm{MeV} \mathrm{H}$ implantation which was chosen to provide a higher displacement damage within the epilayer than 0.6 $\mathrm{MeV} \mathrm{H}$ ions. The sample surface normal was tilted by $7^{\circ}$ with respect to the ion beam incidence direction in order to minimize channeling effects during implantation. The sheet resistance $\left(R_{S}\right)$ of an epilayer was measured in situ after each dose step using a Keithley 617 electrometer. Postirradiation isochronal rapid thermal annealing (RTA) cycles were carried out in the temperature range from 100 up to $700{ }^{\circ} \mathrm{C}$ for $60 \mathrm{~s}$ in an Ar ambient. The variation of sheet resistance of selected samples implanted at room temperature was also measured at different temperatures in order to determine the activation energy for the implant isolation.

\section{RESULTS AND DISCUSSION}

\section{A. Effect of ion species}

Figure 1(a) illustrates the change in sheet resistance, $R_{s}$, with the cumulative dose for $0.6 \mathrm{MeV} \mathrm{H}$ (closed triangle), $0.1 \mathrm{MeV} \mathrm{H}$ (open triangle), $2 \mathrm{MeV} \mathrm{Li} \mathrm{(closed} \mathrm{diamond),} 2$ $\mathrm{MeV} \mathrm{C}$ (closed circle), and 1.2 MeV O ions (closed square). All layers were doped to $4.5 \times 10^{17} \mathrm{Zn} / \mathrm{cm}^{3}$ and implantation was performed at $20^{\circ} \mathrm{C}$ using the ion fluxes given in Table I. Each curve in Fig. 1(a) follows the same general shape that is typical for implant isolation. At the low doses, $R_{s}$ remains relatively unaffected, while it increases abruptly due to free carrier trapping at defect levels and mobility degradation with the increasing dose. The sheet resistance reaches a narrow plateau when the concentration of deep levels is of the same magnitude as the free hole concentration, and is determined by the sheet resistance of the underlying SI GaAs substrate. ${ }^{1,2,7-10,15,16}$ Any further increase in dose beyond this plateau results in the monotonic decrease in $R_{s}$ (closed circles) because of hopping conduction of holes between closely spaced defect potential wells. ${ }^{18}$ Following Ref. 15, a threshold dose for isolation, $D_{\text {th }}$, can be defined as the ion dose at which $R_{s}$ reaches its maximum value [typically $\left.(2-4) \times 10^{9} \Omega / \mathrm{sq}\right]$.

If the data points for $0.1 \mathrm{MeV} \mathrm{H}$ ions are neglected, Fig. 1(a) reveals that the ion dose required for electrical isolation decreases with the increasing ion mass. The exact nature of 


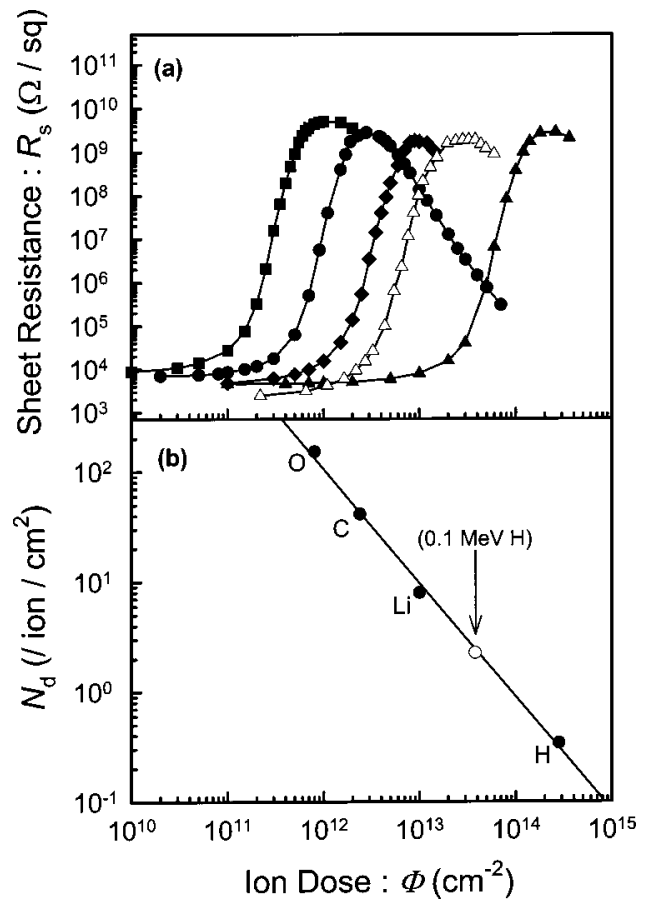

FIG. 1. (a) Dose dependence of sheet resistance, $R_{s}$, for $0.6 \mathrm{MeV} \mathrm{H}$ (closed triangle), $0.1 \mathrm{MeV} \mathrm{H}$ (open triangle), $2 \mathrm{MeV} \mathrm{Li} \mathrm{(closed} \mathrm{diamond),} 2 \mathrm{MeV} \mathrm{C}$ (closed circle), and 1.2 MeV O ions (closed square). Epilayers were doped to $4.5 \times 10^{17} \mathrm{Zn} / \mathrm{cm}^{3}$ and implanted at $20^{\circ} \mathrm{C}$ using the ion fluxes given in Table I. (b) Plot of total number of replacements, $N_{d}$, as a function of the threshold dose for isolation as per (a). The solid line through data points is the least squares fitting with slope $(-1.03 \pm 0.02)$.

this apparent ion mass dependence is better understood by plotting the total number of replacements, $N_{d}$ (i.e., antisite defects) created within the $500 \mathrm{~nm}$ thick epilayer as a function of the threshold ion dose for electrical isolation as depicted in Fig. 1(b). The solid line in Fig. 1(b) represents the least squares fit through the data points and has a slope of $(-1.03 \pm 0.02)$. Although we have used the values of replacement collisions as obtained from TRIM simulations, ${ }^{17}$ it is worth noting that the slope of the linear regression in Fig. 1(b) would remain unity if the total number of vacancies had been used instead of total replacements. This is because the vacancies are precursors for the creation of antisite defects. The choice of the total number of replacements stems partially from the recent discussion of Boudinov et al. ${ }^{16}$ that the defect responsible for the isolation in $p$-type GaAs is the double-donor arsenic-antisite, $\mathrm{As}_{\mathrm{Ga}}$, and the results discussed below. By including the data point for $0.1 \mathrm{MeV} \mathrm{H}$ ions in Fig. 1(b) (see point marked by arrow), it becomes clear that the isolation process does not depend solely on the ion mass, but rather on the total number of defects created by the implanted species. The straight line in Fig. 1(b), therefore, reveals a universal curve over the wide range of ion species used here, suggesting that the isolation process scales close-to-inversely with the number of replacements created in the implanted layer.

\section{B. Effect of doping concentration}

Since it is desirable to have an estimate of the free carrier removal process during isolation for device fabrication

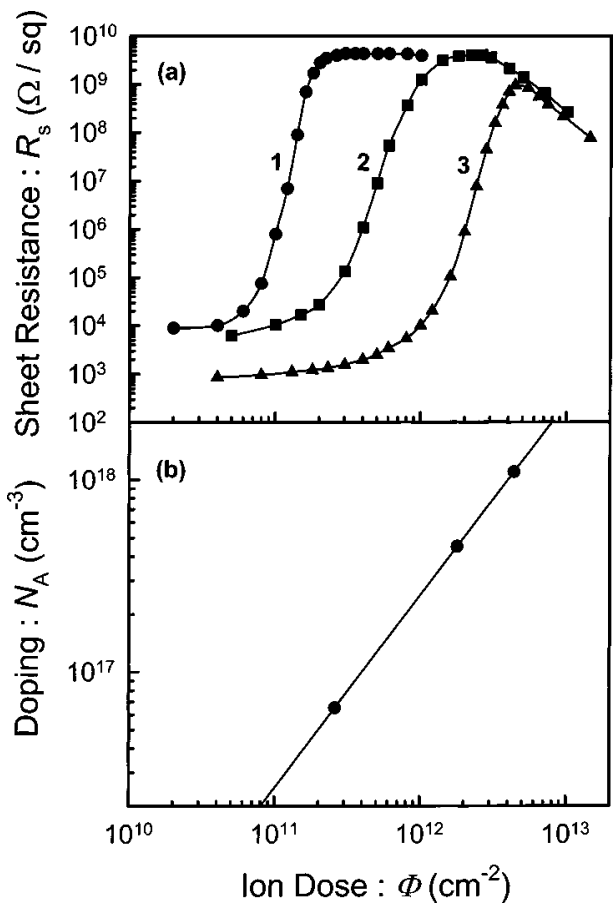

FIG. 2. (a) Evolution of sheet resistance of samples doped with 9 $\times 10^{16} \mathrm{Zn} / \mathrm{cm}^{3} \quad$ (curve 1), $4.5 \times 10^{17} \mathrm{Zn} / \mathrm{cm}^{3} \quad$ (curve 2), or 1.8 $\times 10^{18} \mathrm{Zn} / \mathrm{cm}^{3}$ (curve 3) following $2 \mathrm{MeV} \mathrm{C}$ ion implantation at $20{ }^{\circ} \mathrm{C}$, and (b) the variation of free hole concentration, $N_{A}$, in doped epilayers as a function of the threshold ion dose for isolation obtained from (a). The solid line is the least squares fit through the data points and has a slope of unity.

purposes, investigating the effect of free carrier concentration on the isolation process is crucial. Figure 2(a) illustrates the evolution of sheet resistance of samples doped with 9 $\times 10^{16} \mathrm{Zn} / \mathrm{cm}^{3}$ (curve 1), $4.5 \times 10^{17} \mathrm{Zn} / \mathrm{cm}^{3}$ (curve 2), or $1.8 \times 10^{18} \mathrm{Zn} / \mathrm{cm}^{3}$ (curve 3) following $2 \mathrm{MeV} \mathrm{C}$ ion implantation at $20{ }^{\circ} \mathrm{C}$. The curves exhibit the similar dose dependence except that the ion dose required to achieve the maximum $R_{s}$ is shifted to the higher doses with the increasing free hole concentration. This is clearly illustrated in Fig. 2(b) which shows the variation of free hole concentration, $N_{A}$, in doped epilayers as a function of the threshold ion dose for isolation. The solid line is the least squares fit through the data points and has a slope of unity. The threshold dose for isolation has been defined here as the ion dose required to achieve a $R_{s} \sim 10^{9} \Omega / \mathrm{sq}$. By converting the free carrier concentration to its equivalent sheet concentration, one is able to determine from Fig. 2(b) that an average number of eight carbon ions with energy of $2 \mathrm{MeV}$ are required to compensate 100 holes. These numbers, therefore, enable us to estimate the threshold dose required for the electrical isolation of any $\mathrm{Zn}$-doped layer using $2 \mathrm{MeV} \mathrm{C}$ ions. One observation worth noting in Fig. 2(a) is that the peak sheet resistance that can be reached for the highest doped sample is reduced, in addition to the disappearance of the plateau exhibiting maximum $R_{s}$. This feature can be expected for highly doped samples when hopping conduction becomes a limiting factor for achieving the highest $R_{S}$ due to the high ion doses needed for isolation. 


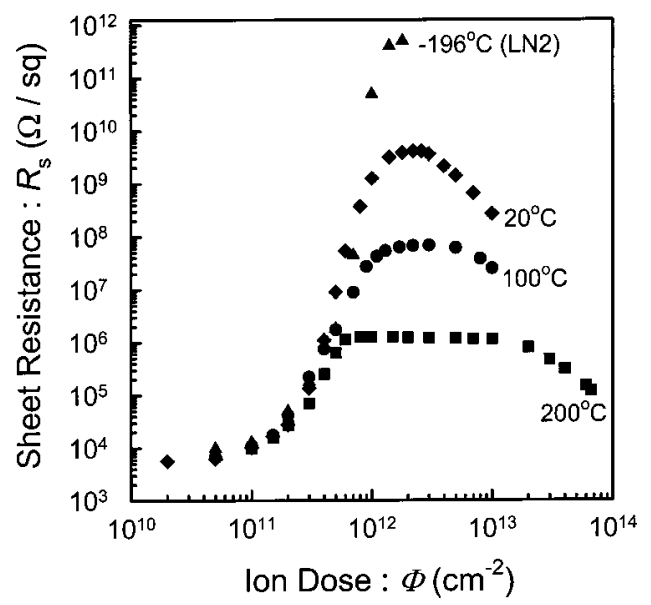

FIG. 3. Evolution of $R_{s}$ for GaAs samples doped with $4.5 \times 10^{17} \mathrm{Zn} / \mathrm{cm}^{3}$ and implanted with $2 \mathrm{MeV} \mathrm{C}$ ions at temperatures ranging from -196 to $200{ }^{\circ} \mathrm{C}$.

\section{Effect of substrate temperature}

It is well established that the dynamic annealing of point defects in GaAs depends strongly on the implantation temperature. ${ }^{10,19}$ Furthermore, isochronal annealing studies of point defects created in electron- and $\gamma$-irradiated GaAs at fairly low doses anneal in three well defined stages between 235 and $500 \mathrm{~K}$ (i.e., $-38-227^{\circ} \mathrm{C}$ ). ${ }^{20-22}$ On the other hand, the defects created in GaAs by ion implantation to moderate doses $\left(<1 \times 10^{14} \mathrm{~cm}^{-2}\right)$ anneal predominantly at the $225^{\circ} \mathrm{C}$ (stage III) and $400{ }^{\circ} \mathrm{C}$ (stage IV) characteristic stages, with the lower annealing temperature related to the mobility of individual point defects, such as the arsenic interstitial, $\mathrm{As}_{i}$, or arsenic vacancy, $V_{\mathrm{As}} .{ }^{20,23,24}$ Yet another stage in the vicinity of $600-700{ }^{\circ} \mathrm{C}$ has been observed for defect annealing in high dose ion implanted GaAs. ${ }^{20}$ This particular annealing stage can be related to the removal of defect clusters or extended defects, which coincides well with the second stage of the solid phase epitaxial regrowth of amorphous GaAs produced by ion implantation. ${ }^{20,25,26}$

With this general guideline for defect annealing in mind, an investigation of the effect of substrate temperature during implantation is important to understand defect-related isolation mechanisms. Such experiments are able to provide insight into the type of defects (i.e., point defects versus defect clusters) responsible for the electrical isolation of $p$-GaAs. It is also worth noting here that all the previous studies on the implantation isolation of $p$-GaAs have, to the best of our knowledge, been performed at room temperature. ${ }^{14-16}$ Figure 3 illustrates the evolution of $R_{s}$ for GaAs samples doped with $4.5 \times 10^{17} \mathrm{Zn} / \mathrm{cm}^{3}$ and implanted with $2 \mathrm{MeV} \mathrm{C}$ ions at temperatures ranging from -196 to $200{ }^{\circ} \mathrm{C}$. The reduction in the maximum value of $R_{S}$ that is reached with the increasing substrate temperature during implantation can be attributed to the increased ionization of holes trapped at defect centers at the higher temperatures. Furthermore, one is cautioned regarding the absolute value of $R_{s}$ reached at $-196^{\circ} \mathrm{C}$ because of the detection limit of our electrometer and contributions from the parasitic resistances of the experimental setup. The curves in Fig. 3 clearly show that $R_{s}$ starts to increase at the same dose of $\sim 2 \times 10^{11} \mathrm{C} / \mathrm{cm}^{2}$ irrespective of the substrate temperature during implantation. Furthermore, the saturation in $R_{s}$ appears to occur in the same ion dose regime for all the implantation temperatures, i.e., $\sim(2-4)$ $\times 10^{12} \mathrm{~cm}^{-2}$. The results shown in Fig. 3 are qualitatively similar to those reported previously for implant isolation of $n$-type GaAs when no significant influence of substrate temperature on the isolation process was observed in the range $-100-220^{\circ} \mathrm{C}^{10}$ It can, therefore, be concluded from the above discussion that the defects responsible for the stability of implant isolation in $p$-GaAs are not point defects (i.e., Frenkel-type defects, such as vacancies and interstitials) but rather defect complexes that have a higher thermal stability.

\section{Thermal stability and stages in the buildup of electrical isolation}

The thermal stability of implant isolation is a critical issue to consider in device applications. In particular, maximizing the thermal stability of the implant isolation is desirable. The previous studies that have investigated the thermal stability of implant isolation in $p$-GaAs have used either only one ion species ${ }^{15,16}$ or multiple ion implantation schemes using different ions to create a uniform defect profile in the conducting $p$-GaAs layer. ${ }^{14}$ In the latter case, the difference between the thermal stability between $\mathrm{H}$ and $\mathrm{F}$ implantation isolation was attributed to an ion mass effect. In this section, we provide a detailed discussion of the effects of ion dose and ion species on the thermal stability of implantation isolation. Although we are not able to correlate the stability of implantation isolation with any particular defect(s), this study does provide much insight into the types of defects that contribute to the isolation based on their annealing behavior. Further information on the buildup of a stable isolation with the increasing ion dose is also obtained through temperature dependent measurements of the sheet resistance of implanted samples both before and after annealing.

Figure 4(a) illustrates the thermal stability of isolation for layers implanted with $2 \mathrm{MeV} \mathrm{C}$ ions at $20^{\circ} \mathrm{C}$ to doses ranging from $0.5 D_{\text {th }}\left(1.2 \times 10^{12} \mathrm{~cm}^{-2}\right)$ to $29.17 D_{\text {th }}(7$ $\left.\times 10^{13} \mathrm{~cm}^{-2}\right)$. The epilayers were doped with 4.5 $\times 10^{17} \mathrm{Zn} / \mathrm{cm}^{3}$. Each data point on the curves in Fig. 4(a) is comprised of the cumulative isochronal annealing sequence for $60 \mathrm{~s}$ periods at the lower RTA temperatures. It is obvious from Fig. 4(a) that the thermal stability of isolation depends critically on the ion dose. An increase in $R_{s}$ is observed for the sample bombarded to $0.5 D_{\text {th }}$ in the temperature range $20-250{ }^{\circ} \mathrm{C}$. This effect is similar to that observed in a previous report of the thermal stability of isolation in $\mathrm{H}$ implanted $p$-GaAs. ${ }^{15}$ Two possible scenarios for this increase in $R_{s}$ during annealing between 20 and $250^{\circ} \mathrm{C}$ can be envisaged, namely (a) the formation of more efficient hole trapping centers due to interaction of point-like defects and/or (b) the increase in the concentration of existing hole trapping centers. Indeed, a previous investigation of the annealing of defects created in electron irradiated $p$-GaAs epilayers has demonstrated that a hole trap P1 $\left(E_{V}+0.36 \mathrm{eV} ; \sigma_{a}=6.9\right.$ $\times 10^{-15} \mathrm{~cm}^{2}$ ) is created upon annealing at the expense of electron traps that are produced initially by primary colli- 


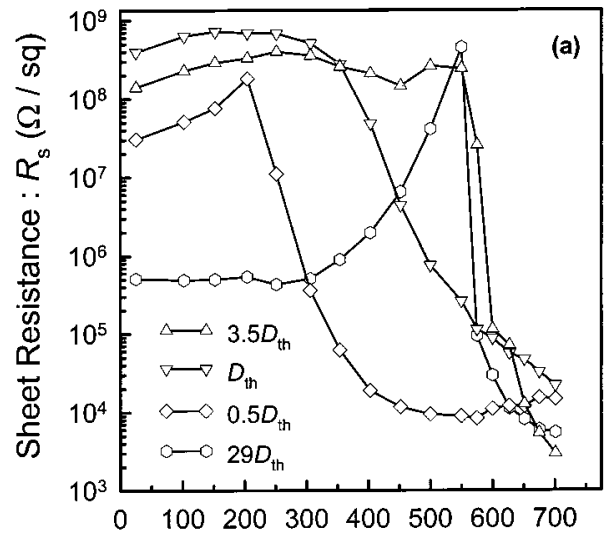

Annealing Temperature : $T\left({ }^{\circ} \mathrm{C}\right)$

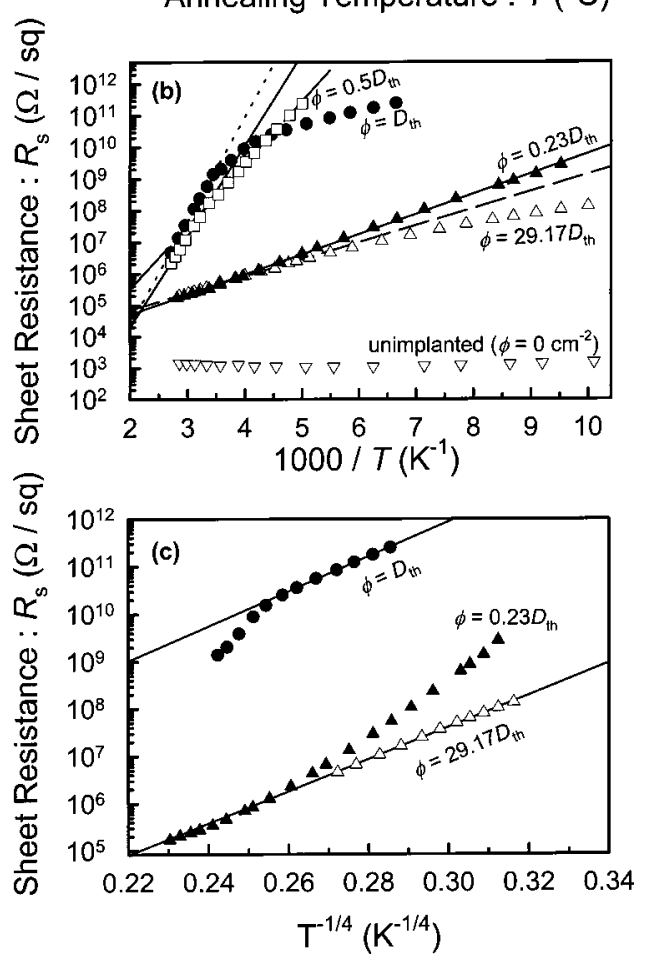

FIG. 4. (a) Isochronal annealing stability of sheet resistance for layers implanted with $2 \mathrm{MeV} \mathrm{C}$ ions at $20^{\circ} \mathrm{C}$ to doses ranging from $0.5 D_{\text {th }}(1.2$ $\left.\times 10^{12} \mathrm{~cm}^{-2}\right)$ to $29.17 D_{\text {th }}\left(7 \times 10^{13} \mathrm{~cm}^{-2}\right)$. The epilayers were doped with $4.5 \times 10^{17} \mathrm{Zn} / \mathrm{cm}^{3}$. (b) Arrhenius-like dependence of $\ln \left(R_{s}\right)$ vs $1 / T$ for the different ion doses marked on the curves, and (c) temperature variation of sheet resistance of C-implanted layers to doses marked on the curves plotted as $\ln \left(R_{s}\right)$ versus $T^{1 / 4}$.

sions, and its concentration increased upon annealing up to $\sim 280^{\circ} \mathrm{C} .{ }^{22}$ An apparent discrepancy between the results in Fig. 4(a) discussed thus far and those shown in Fig. 3 (i.e., no significant effect of substrate temperature during implantation) may transpire. However, the reader is cautioned that defects created during implantation at $200{ }^{\circ} \mathrm{C}$ may well be different to defects present in samples implanted at room temperature and subsequently annealed at $200{ }^{\circ} \mathrm{C}$. We plan to perform detailed studies of defects created in $p$-GaAs at different temperatures and also to investigate their postimplantation annealing kinetics in order to resolve this issue. Annealing of the layer implanted to $0.5 D_{\text {th }}$ above $205^{\circ} \mathrm{C}$ results in the gradual recovery of free carriers most probably due to annealing of point-like defects in the characteristic stage III annealing of defects in $p$-GaAs. ${ }^{20-24}$

The sheet resistance of the layer implanted to $D_{\text {th }}$ is thermally stable up to $\sim 350^{\circ} \mathrm{C}$ above which it decreases monotonically with the increasing temperature. It becomes clear from the above discussion that the defects responsible for the electrical isolation of the sample bombarded to $D_{\text {th }}$ anneal in the characteristic stage IV typical of samples implanted to moderate doses. ${ }^{20,23,24}$ The defects responsible for free carrier trapping in the sample implanted to $D_{\text {th }}$ are most probably defect pairs, resulting from the interactions of vacancies, interstitials, and impurities, rather than simple isolated defects. $^{21}$ Bombarding the $p$-GaAs epilayer to $3.5 D_{\text {th }}$ enhances the thermal stability of isolation up to $550{ }^{\circ} \mathrm{C}$, which is sufficient for device application purposes. The initial increase in sheet resistance of the sample implanted to $29.17 D_{\text {th }}$ with the increasing temperature results from the recovery of hopping conduction. Defects are removed with the increasing temperature so that the spatial distribution of defect potential wells that trap free carriers decreases. The maximum $R_{s}$ is reached at $550^{\circ} \mathrm{C}$ when hopping conduction is completely eliminated, while the concentration of defects responsible for hole trapping is of the same order of magnitude as the initial free carrier concentration. Similar to the case of $3.5 D_{\text {th }}$ these defects are removed by annealing above $550{ }^{\circ} \mathrm{C}$, which would correspond to the annealing of defect clusters or defect complexes in GaAs. ${ }^{20,21}$ A recent study has proposed that the double donor $\mathrm{As}_{\mathrm{Ga}}^{+/++}$(defect EL2 with an energy position of $E_{V}+0.54 \mathrm{eV}$ in the band gap) could be a suitable candidate for the defect responsible for the stability of isolation in $p$-GaAs. ${ }^{16,27}$ Indeed, it has been reported that the EL2 introduced in neutron-irradiated, plastically deformed, or low-temperature GaAs grown by molecular beam epitaxy, anneal in the temperature range of $450-600{ }^{\circ} \mathrm{C}$. $^{28,29}$ More recently, Tan et al. ${ }^{30}$ have shown that the excess EL2 created by proton implantation of GaAs epilayers grown by MOCVD was stable only up to $500{ }^{\circ} \mathrm{C}$, which correlates very well with the temperature window within which the isolation of C-implanted $p$-GaAs becomes unstable [Fig. 4(a)]. This discussion forms the basis for using values of replacements rather than vacancies in Fig. 1(b). These results are in good agreement with those depicted in Fig. 3, when it was concluded that the defects responsible for stable isolation could not be point-like. We can, therefore, conclude from Fig. 4(a) that an ion dose of $3.5 D_{\text {th }}$ is sufficient for creating a stable isolation in $p$-GaAs. An ion dose larger than $4 D_{\text {th }}$ required for the stable isolation in a previous study could be attributed to the nonuniform defect profile generated by low-energy protons in the $p$-GaAs layer. ${ }^{15}$

Further insight into the buildup of stable electrical isolation with the increasing ion dose is obtained by measuring the temperature dependence of $R_{s}$. Figure 4(b) illustrates the Arrhenius-like dependence of $R_{s}$ on the inverse of measurement temperature, $T$, for $p$-GaAs layers $\left(4.5 \times 10^{17} \mathrm{Zn} / \mathrm{cm}^{3}\right)$ implanted to different doses at $20^{\circ} \mathrm{C}$. The data points shown in open inverted triangles correspond to the unimplanted sample, and they do not reveal any striking feature. However, the curve for $0.5 D_{\text {th }}$ (open squares) exhibits two regimes each of which can be well described by least squares 
fits (solid lines). These fits yield activation energies of $(0.39 \pm 0.02 \mathrm{eV})$ and $(0.57 \pm 0.02 \mathrm{eV})$ for the isolation process in the sample implanted to $0.5 D_{\text {th }}$. A comparison with the unimplanted sample and the temperature dependence of the carrier mobility and free carrier concentration in irradiated GaAs clearly shows that two discrete defects are responsible for the isolation in $p$-GaAs implanted to $0.5 D_{\text {th }} \cdot{ }^{31}$ The activation energies should be treated with caution since they consist of two components, namely (a) the ionization energy of holes from trapping centers (i.e., activation energy of deep level), and (b) the activation energy for hole scattering at defect sites (i.e., the mechanism for degradation of hole mobility in implanted samples). The activation energy of the defects can, therefore, be extracted only after establishing the temperature dependence of the hole mobility in implanted samples.

Only one defect is responsible for the isolation [activation energy $(0.13 \pm 0.02 \mathrm{eV})$ of a layer implanted to $0.23 D_{\text {th }}$ [closed triangles in Fig. 4(b)]. This activation energy is most probably related to the ionization of holes to the valence band, as opposed to thermally activated interdefect hopping conduction. A conducting $p$-GaAs layer implanted also to $D_{\text {th }}$ exhibits two temperature regimes [closed circles in Fig. 4(b)], except that an Arrhenius-like dependence of $\ln \left(R_{S}\right)$ on $1 / T$ is observed only for $T>280 \mathrm{~K}$. The dotted line is the linear regression through these data points, which yields an activation energy of $(0.65 \pm 0.03 \mathrm{eV})$ for the process. For $T$ $<280 \mathrm{~K}$, the data points are well described by a linear relationship between $\ln \left(R_{S}\right)$ and $T^{-1 / 4}$ [Fig. 4(c)], which is characteristic of the hopping conduction mechanism between localized defect states near the Fermi level as proposed by Mott. ${ }^{18,32,33}$ The $p$-type layer implanted to the highest dose of $29.17 D_{\text {th }}$ is heavily damaged as demonstrated by the hopping conduction mechanism in it [Figs. 4(b) and 4(c)]. In the higher temperature $(T>200 \mathrm{~K})$ range, the slope of the Arrhenius plot [Fig. 4(b)] yields an activation energy of $(0.11 \pm 0.01 \mathrm{eV})$, which is in good agreement with the value obtained for Te-implanted GaAs to high doses. ${ }^{33}$ At temperatures below $200 \mathrm{~K}$, the $R_{S}$ curve can be better fitted to the Mott relationship for hopping conduction [Fig. 4(c)]. Using Mott's theory, ${ }^{32}$ and the detailed formalism described in Refs. 18 and 33, a value of $\sim 6.5 \mathrm{~nm}$ can be deduced from Figs. 4(b) and 4(c) for the hopping distance in the layer bombarded to $29.17 D_{\mathrm{th}}$. For $T>200 \mathrm{~K}$, hopping of carriers takes place between the nearest neighbors (i.e., defect sites within $\sim 6.5 \mathrm{~nm}$ to each other) leading to the Arrhenius-like dependence of $\ln \left(R_{s}\right)$ versus $1 / T$, whereas holes can tunnel to more distant trapping sites at the lower temperatures according to $\ln \left(R_{s}\right) \propto T^{-1 / 4}$. ${ }^{33}$ Figure 4(c) also features the data points for $0.23 D_{\text {th }}$ in order to confirm the absence of a hopping conduction mechanism in the low-dose ion implantation regime. The stages of the buildup of implantation isolation with increasing ion dose are evident from Fig. 4. Isolated point defects which are thermally stable up to $\sim 205^{\circ} \mathrm{C}$ are responsible for free carrier trapping at the low ion doses, while point defect pairs (stable up to $\sim 400^{\circ} \mathrm{C}$ ) are likely to cause isolation in the intermediate dose regime.

We now turn to the influence of the ion species on the thermal stability of isolation in $p$-GaAs. Figure 5(a) illus-
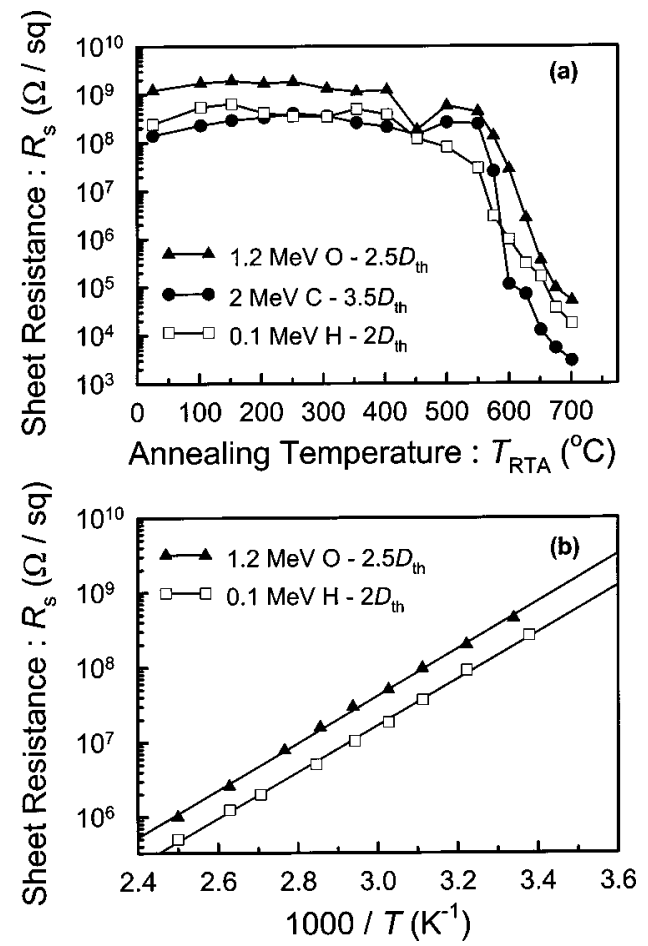

FIG. 5. (a) Evolution of sheet resistance for H- (open squares), C- (closed circles), and $\mathrm{O}$ - implanted (closed triangles) $p$-GaAs resistors at $20^{\circ} \mathrm{C}$ to 2-3.5 times their corresponding threshold doses, $D_{\text {th }}$, and (b) the variation of $\ln \left(R_{s}\right)$ as a function of $1 / T$ for $\mathrm{H}$ - and O-implanted resistors yielding activation energies of $(0.62 \pm 0.03 \mathrm{eV})$ and $(0.63 \pm 0.03 \mathrm{eV})$, respectively. The temperature dependent measurements were made after samples were annealed at $450{ }^{\circ} \mathrm{C}$.

trates the change of $R_{s}$ for $\mathrm{H}$ - (open squares), C- (closed circles), and O-implanted (closed triangles) $p$-GaAs resistors to 2-3.5 times their corresponding threshold doses, $D_{\text {th }}$. All three samples implanted at $20^{\circ} \mathrm{C}$ demonstrate the similar thermal stability of isolation. It is pointed out here that the similar annealing behavior was observed for samples implanted at $-196,100$, or $200^{\circ} \mathrm{C}$, which was further evidence that the implantation temperature did not play a significant role in the isolation process. We show in Fig. 5(b) the variation of $\ln \left(R_{s}\right)$ as a function of $1 / T$ for the $\mathrm{H}$ - and O-implanted samples (i.e., the lightest and heaviest ion species used in this study) for temperatures above $290 \mathrm{~K}$ when the trapped carriers become thermally activated. The measurements were made after the samples were annealed at $450{ }^{\circ} \mathrm{C}$. The least squares fits through the data points shown as solid lines in Fig. 5(b) yield activation energies of $(0.62 \pm 0.03 \mathrm{eV})$ and $(0.63 \pm 0.03 \mathrm{eV})$ for the isolation process by $\mathrm{H}$ and $\mathrm{O}$ implantation, respectively. It is timely here to remind the reader that an activation energy of $(0.65 \pm 0.03 \mathrm{eV})$ was obtained from Fig. 4(b) for $\mathrm{C}$ implantation. The similar activation energies for the different ions indicate the absence of an ion mass effect on the isolation process, which is supported by the earlier discussion of the results in Fig. 1. The crux of the implant isolation process is the nuclear energy deposited in elastic recoils within the conducting layer. In a previous study of the long-term thermal stability of implant-isolated $p$-GaAs, Ren et al. ${ }^{14}$ extracted different activation energies for the isolation of $\mathrm{H}$ - and $\mathrm{F}$-implanted $p$-GaAs. Following 
these results, it was concluded that the ion mass played a role in the thermal stability of isolation. The discrepancy between the results reported here and those in Ref. 14 can be explained by the different implantation schemes employed in the two studies. We believe that the types and concentration of defects created by the multiple implantations in Ref. 14 were different for $\mathrm{H}$ - and F-ion implantation, as opposed to the single ion implantation scheme used here to create a uniform defect profile within the conducting $p$-GaAs layers. The absence of an ion mass effect has previously been reported for the implantation isolation of $n$-GaAs layers. ${ }^{8}$

\section{CONCLUSION}

In summary, the electrical isolation of $p$-GaAs epilayers grown by metalorganic chemical vapor deposition and doped to different concentrations of $\mathrm{Zn}$ were used to study the formation of implantation isolation for a wide range of ions. Results have clearly demonstrated that the isolation process was determined predominantly by the nuclear energy deposited in elastic recoils during implantation. This is evidenced by the similar activation energy for isolation obtained for both $\mathrm{H}$ and $\mathrm{O}$ ion implantation, and the similar thermal stability of isolation of $p$-GaAs layers bombarded with different ions during postimplantation isochronal annealing. By performing $2 \mathrm{MeV} \mathrm{C}$ ion implantation of GaAs epilayers doped with different $\mathrm{Zn}$ concentrations, it was found that eight carbon ions are required to compensate 100 holes. The buildup of isolation with the dose of $2 \mathrm{MeV} \mathrm{C}$ ions follows the creation of (i) point defects for $\phi<0.5 D_{\text {th }}$, (ii) defect pairs for $\phi=D_{\text {th }}$, and (iii) defect clusters or complexes (e.g., $\mathrm{As}_{\mathrm{Ga}}$ ) that are stable at $550{ }^{\circ} \mathrm{C}$ for the higher ion doses. The thermal stability of isolation in these three ion dose regimes correspond well with the characteristic annealing stages of defects in ion implanted GaAs previously reported in the literature. The substrate temperature $\left(-196-200^{\circ} \mathrm{C}\right)$ during implantation did not have any bearing on the isolation process, demonstrating that point-like defects do not contribute to the stability of implant isolation of $p$-GaAs.

\section{ACKNOWLEDGMENT}

P.N.K.D. acknowledges the Australian Research Council for financial support.
${ }^{1}$ S. J. Pearton, Mater. Sci. Rep. 4, 313 (1990).

${ }^{2}$ S. J. Pearton, Int. J. Mod. Phys. B 7, 4687 (1993).

${ }^{3}$ K. Berthold, A. F. J. Levi, S. J. Pearton, R. J. Malik, W. Y. Jan, and J. E. Cunningham, Appl. Phys. Lett. 55, 1382 (1989).

${ }^{4}$ T. E. Sale, in Vertical Cavity Surface Emitting Lasers (Research Studies, Exeter, 1995), p. 18.

${ }^{5}$ G. Dang, B. Luo, F. Ren, W. S. Hobson, J. Lopata, S. N. G. Chu, and S. J. Pearton, Electrochem. Solid-State Lett. 4, G112 (2001).

${ }^{6}$ M. Y. A. Raja, Y. Cao, G. H. Cooper, A. S. Al-Dwayyan, and C. X. Wang, Opt. Eng. (Bellingham) 41, 704 (2002).

${ }^{7}$ J. P. de Souza, I. Danilov, and H. Boudinov, Appl. Phys. Lett. 68, 535 (1996).

${ }^{8}$ J. P. de Souza, I. Danilov, and H. Boudinov, J. Appl. Phys. 81, 650 (1997).

${ }^{9}$ J. P. de Souza, I. Danilov, and H. Boudinov, Nucl. Instrum. Methods Phys. Res. B 122, 51 (1997).

${ }^{10}$ J. P. de Souza, I. Danilov, and H. Boudinov, J. Appl. Phys. 84, 4757 (1998).

${ }^{11}$ S. Ahmed, R. Gwilliam, and B. J. Sealy, Semicond. Sci. Technol. 16, L28 (2001).

${ }^{12}$ S. Ahmed, R. Gwilliam, and B. J. Sealy, Semicond. Sci. Technol. 16, L64 (2001).

${ }^{13}$ S. Ahmed, R. Gwilliam, and B. J. Sealy, Nucl. Instrum. Methods Phys. Res. B 188, 196 (2002).

${ }^{14}$ F. Ren, S. J. Pearton, C. R. Abernathy, P. W. Wisk, T. R. Fullowan, J. R. Lothian, and R. Esagui, Semicond. Sci. Technol. 8, 605 (1993).

${ }^{15}$ J. P. de Souza, I. Danilov, and H. Boudinov, Radiat. Eff. Defects Solids 147, 109 (1998)

${ }^{16}$ H. Boudinov, A. V. P. Coelho, and J. P. de Souza, J. Appl. Phys. 91, 6585 (2002).

${ }^{17}$ J. F. Ziegler, J. P. Biersack, and U. Littmark, The Stopping and Range of Ions in Solids (Pergamon, Oxford, 1985), Vol. 1.

${ }^{18}$ Y. Kato, T. Shimada, Y. Shiraki, and K. F. Komatsubara, J. Appl. Phys. 45, 1044 (1974)

${ }^{19}$ T. E. Hayes and O. W. Holland, Appl. Phys. Lett. 59, 452 (1991).

${ }^{20}$ D. V. Lang, Inst. Phys. Conf. Ser. 31, 70 (1977).

${ }^{21}$ D. Pons and J. C. Bourgoin, J. Phys. C 18, 3839 (1985).

${ }^{22}$ D. Pons, A. Mircea, and J. Bourgoin, J. Appl. Phys. 51, 4150 (1980).

${ }^{23}$ J. C. Bourgoin, H. J. von Bardeleben, and D. Stiévenard, J. Appl. Phys. 64, R65 (1988).

${ }^{24}$ D. Stiévenard, X. Boddaert, and J. C. Bourgoin, Phys. Rev. B 34, 4048 (1986).

${ }^{25}$ J. S. Williams, in Laser Annealing of Semiconductors, edited by J. M. Poate and J. W. Mayer (Academic, New York, 1982), p. 383, and references therein.

${ }^{26}$ S. S. Kular, B. J. Sealy, K. G. Stephens, D. K. Sadana, and G. R. Booker, Solid-State Electron. 23, 831 (1980).

${ }^{27}$ J. Lagowski, D. G. Lin, T.-P. Chen, M. Skowronski, and H. C. Gatos, Appl. Phys. Lett. 47, 929 (1985).

${ }^{28}$ R. Wörner, U. Kaufman, and J. Schneider, Appl. Phys. Lett. 40, 141 (1982).

${ }^{29}$ M. Kaminska and E. R. Weber, Mater. Sci. Forum 83-87, 1033 (1992).

${ }^{30}$ H. H. Tan, J. S. Williams, and C. Jagadish, J. Appl. Phys. 78, 1481 (1995).

${ }^{31}$ D. C. Look, in Electrical Characterization of GaAs Materials and Devices (Wiley, Chichester, 1992), pp. 69-131.

${ }^{32}$ N. F. Mott, J. Non-Cryst. Solids 1, 1 (1968).

${ }^{33}$ B. K. Shin, J. Appl. Phys. 47, 3612 (1976). 